\title{
A novel ultrasound-based technique to establish a correlation between disease activity and local carotid stiffness parameters in rheumatoid arthritis
}

\author{
Ayşe Nur Şirin Özcan¹, Abdullah Nabi Aslan², Özlem Ünal1, Karabekir Ercan¹, \\ Orhan Küçükşahin ${ }^{3}$
}

${ }^{1}$ Atatürk Education and Research Hospital, Department of Radiology, ${ }^{2}$ Atatürk Education and Research Hospital, Department of Cardiology, ${ }^{3}$ Yıldırım Beyazit University, Medical Faculty, Department of Rheumatology, Ankara, Turkey

\begin{abstract}
Aim: Cardiovascular (CV) disease is the reason for most mortality cases in RA and cannot be explained only by the presence of traditional CV risk factors. In this study, we aimed to investigate the relationship between local carotid stiffness (CS) parameters measured by a novel ultrasound method and inflammatory disease activity in rheumatoid arthritis (RA) patients. Material and methods: The study was conducted with 70 RA patients and 35 control subjects. According to their disease activity score (DAS-28), the RA patients were classified into active RA ( $\mathrm{n}=36$; DAS-28 > 3.2) and inactive RA ( $\mathrm{n}=34$; DAS-28 $\leq 3.2$ ) groups. A novel non-invasive echo-tracking system was used to measure carotid intima-media thickness (C-IMT), diameter, pulsatile strain, distensibility, and carotid pulse wave velocity (PWV) on 128 sites of the common carotid artery. Erythrocyte sedimentation rate (ESR) and C-reactive protein (CRP) levels were also determined. Results: Carotid PWV and IMT were significantly higher in the active RA patients $(8.20 \pm 1.47 \mathrm{~m} / \mathrm{s}$ and $6.88 \pm 1.50 \mathrm{~mm}$, respectively) compared to the inactive group $(6.06 \pm 1.21 \mathrm{~mm}$ and $7.32 \pm 1.19 \mathrm{~m} / \mathrm{s}$, respectively) and the control subjects $(0.68 \pm 0.12 \mathrm{~mm}$ and $6.41 \pm 0.98$, respectively). In all RA patients, a statistically significant correlation was found between carotid PWV and age $(\mathrm{r}=0.435, \mathrm{p}<0.001)$, ESR $(\mathrm{r}=0.257, \mathrm{p}=0.033)$, and DAS-28 $(\mathrm{r}=0.314, \mathrm{p}=0.009)$. According to the multivariate logistic regression analysis, age, DAS-28, and ESR were independent predictors of CS. Conclusion: A strong correlation was found between disease activity and local CS parameters in patients with RA. We also demonstrated that both active and inactive RA patients showed increased PWV values compared with the control subjects. This easily applicable and previously confirmed method can be used in future to assess cardiovascular risk in broad study populations from different risk groups.
\end{abstract}

Keywords: echotracking system; rheumatoid arthritis; local carotid stiffness; intima-media thickness

\section{Introduction}

Rheumatoid arthritis (RA), a chronic inflammatory disease, has a prevalence of $0.5-1 \%$ in Northern Europe and North America [1,2]. Although RA primarily affects the connective tissue, the mortality in RA is related to the increased prevalence of cardiovascular (CV) disease

Received 24.01.2017 Accepted 18.04.2017

Med Ultrason

2017, Vol. 19, No 3, 288-294

Corresponding author: Ayșe Nur Şirin Özcan M.D

Ankara Ataturk Education and Research Hospital Department of Radiology, 06800 Ankara, Turkey

Phone: +905062046018, Fax: +903122912726

E-mail: aysenursirinozcan@gmail.com
[3-5] and therefore several factors independently predict the heightened CV risk observed in patients with RA. Systemic inflammation is one of the important contributors by accelerating various changes in the arterial wall, such as endothelial dysfunction, increased expression of adhesion molecules, smooth muscle proliferation, and aortic stiffening, and directly accelerating the atherosclerotic process and plaque destabilization in RA [6,7]. Endothelial dysfunction can be observed early in the course of atherogenesis and plays an essential role in all the phases of the atherosclerotic process [7]. It is not only caused by traditional risk factors but also other risk factors including inflammatory mediators, treatment, and disease activity [8]. Due to its precursor role in atherosclerosis, endothelial function is 
assessed on different regions of the body and using various methods such as a flow-mediated dilation test, pulse wave velocity (PWV) analysis, and finger plethysmography [9].

Pulse wave velocity (PWV) is the primary parameter to assess arterial stiffness (AS) and refers to the speed at which a pulse wave moves through an arterial segment [10]. As a consequence of atherosclerosis the arteries become stiffer and the PWV increases. The gold standard method to measure PWV is the use of flowmeters or catheter-based pressure probes; however, due to the difficulty of these applications, other methods such as applanation tonometry and Doppler ultrasound are widely preferred [10]. These two techniques measure the global PWV. Pulse waves are detected by an ultrasound transducer or pressure sensitive transducer from two remote locations and the distance is divided by time to obtain PWV. In practice, measurements are usually made from carotid and femoral arteries but there is no consensus in terms of measuring the distance between two arterial segments. In addition to these techniques, a PWV analysis can be performed locally from the common carotid artery (CCA) via an ultrasound system as firstly described by Luo et al [11]. The authors obtained PWV from the spatial-temporal variation of the carotid wall velocities. Recently, a novel method called ultrasound radio-frequency (RF) echo-tracking system has been developed to evaluate local carotid stiffness (CS). The efficiency of this technique was previously confirmed by Giannarelli et al using the applanation tonometry [12]. However, determining an independent predictor in RA remains to be very important because traditional risk factors are not sufficient to explain the subclinical atherosclerosis. Several techniques and parameters have been used to detect the subclinical atherosclerosis. Among these, PWV is one of the most frequently used parameters. Different complicated and expensive techniques have also been employed to evaluate PWV. Determining PWV with ultrasonography is easier and it does not require the use of an additional device.

In the present study, we aimed to identify an independent predictor for subclinical atherosclerosis in RA patients via an easy, feasible and widely applicable technique that can be used in broad study groups and integrated into design prevention schemes and therapeutic approaches. Furthermore, to the best of our knowledge, the present study was the first to investigate the correlation between RA disease activity and local CS using this system.

\section{Materials and methods}

\section{Study population}

Local CS and carotid intima-media thickness (CIMT) were assessed in 70 patients with RA (7 male and 63 female, mean age $52.2 \pm 10.8$ years) and 35 control subjects (4 male and 31 female, mean age 53.5 \pm 6.7 years). The patients were recruited from the rheumatology outpatient clinic of our hospital and the RA diagnosis was made based on the 2010 ACR/EULAR Classification Criteria. The patients were then classified according to their disease activity scores (DAS) into the groups of active RA $(n=36)$ and inactive RA $(n=34)$. DAS was calculated using a DAWN visual DAS-28 calculator. C-reactive protein (CRP) was selected as an additional measure to calculate DAS-28. The patients with a DAS28 score of equal to or higher than 3.2 were included in the active RA group and those that scored under 3.2 were included in the inactive RA group. The exclusion criteria were history of a $\mathrm{CV}$ disease, hypertension, diabetes mellitus, other systemic inflammatory diseases, smoking, and alcohol consumption. For the patients included in the study, the following parameters were recorded: body mass index (BMI), heart rate, disease duration, fasting blood glucose (FBG), blood urea nitrogen (BUN), creatinine, total cholesterol (TC), high-density lipoprotein cholesterol (HDL-C), low-density lipoprotein cholesterol (LDL-C), triglycerides (TG), ESR, CRP levels, estimated glomerular filtration rate (eGFR), and systolic and diastolic blood pressure (BP).

The examinations were performed after taking written consent from each subject, with approval from the local Ethics Committee, and in compliance with national legislation and the Declaration of Helsinki guidelines.

\section{Evaluation of local carotid arterial stiffness}

A high-resolution B-mode ultrasound system (MyLab25 QAS machine; Carotid Studio, ESAOTE, Genova, Italy) with a 7.5-12 MHz linear array transducer was used for carotid examination. Bilateral common carotid arteries were evaluated from $1 \mathrm{~cm}$ beneath carotid bifurcation at the Radiology Department by a radiologist with an 8-year experience. Longitudinal images that demonstrated double lines representing the intima-media layer were used to obtain carotid quality arterial stiffness (QAS) and quality intima-media thickness (QIMT) to determine intima-media thickness and arterial diameter between the systolic and diastolic phases based on the RF reception signal (fig 1) [12]. QAS variations in the arterial diameter during six cardiac cycles were displayed in real time on a graphic giving the operator visual feedback and standard deviation (SD) values to adjust the quality of image before performing the measurement (fig 2). Similar to the QAS measurement, the last six QIMT values were shown on screen and adjusted after several consecutive measurements. The QIMT and QAS mean values for the last six measurements with less than 15 SD were accepted as sufficient in terms of quality. QIMT 


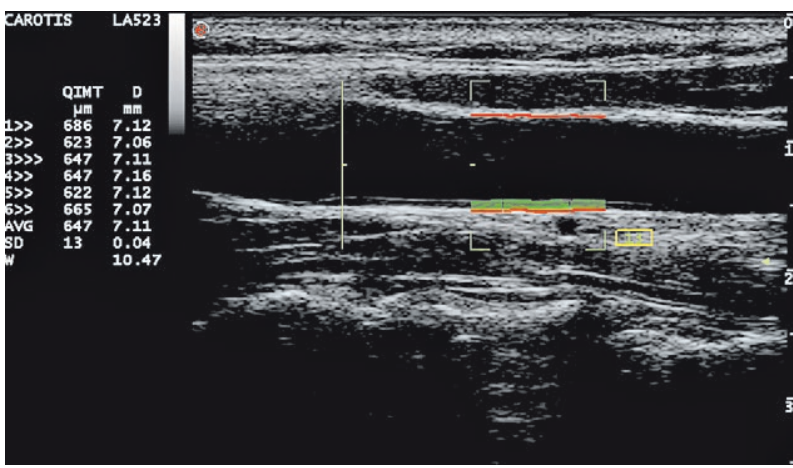

Fig 1. QIMT analysis of the common carotid artery. Green lines correspond to the intima-media layer. Six consecutive measurements were made and a mean value was obtained.

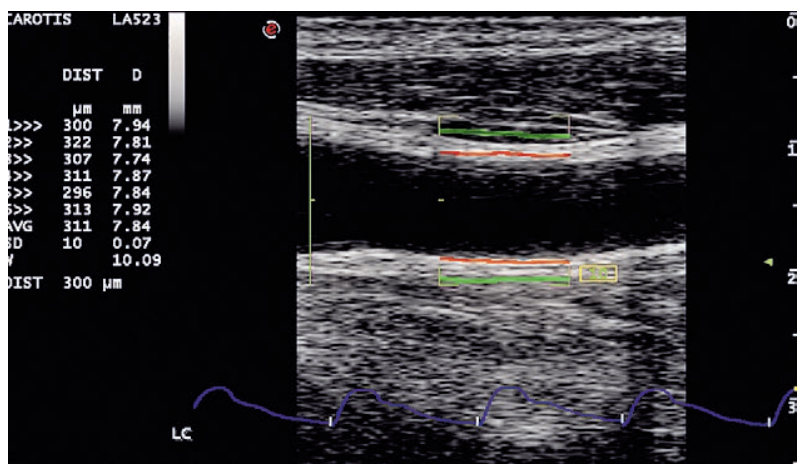

Fig 2. QAS analysis of the common carotid artery. Red and green line measure media-adventitia distance as the carotid diameter. During six cardiac cycle systolic and diastolic vessel displacement detected and calculated carotid distension and also other AS parameters were calculated automatically by using the Moens-Korteweg equation.

Table I. Clinical characteristics of the study population.

\begin{tabular}{|c|c|c|c|c|}
\hline Variables & Controls $(n=35)$ & Inactive RA group $(n=34)$ & Active RA group $(n=36)$ & p value \\
\hline Age (years) & $53.5 \pm 6.7$ & $53.5 \pm 9.3$ & $50.9 \pm 12.0$ & 0.327 \\
\hline Women, n (\%) & $31(88.5)$ & $28(82.3)$ & $35(97.2)$ & 0.039 \\
\hline $\operatorname{BMI}\left(\mathrm{kg} / \mathrm{m}^{2}\right)$ & $28.3 \pm 3.7$ & $29.7 \pm 6.0$ & $27.4 \pm 5.5$ & 0.153 \\
\hline Systolic BP (mmHg) & $131.7 \pm 23.2$ & $128.3 \pm 20.1$ & $128.3 \pm 20.9$ & 0.997 \\
\hline Diastolic BP (mmHg) & $88.7 \pm 14.9$ & $80.6 \pm 17.0$ & $81.3 \pm 15.8$ & 0.873 \\
\hline $\mathrm{MBP}(\mathrm{mmHg})$ & $103.0 \pm 16.5$ & $96.5 \pm 17.2$ & $96.9 \pm 16.7$ & 0.916 \\
\hline Brachial PP (mmHg) & $43.0 \pm 15.7$ & $47.6 \pm 11.7$ & $47.0 \pm 12.3$ & 0.832 \\
\hline Heart rate, bpm & $76.5 \pm 14.0$ & $76.4 \pm 7.8$ & $77.2 \pm 8.1$ & 0.844 \\
\hline Disease duration (years) & NA & $9.4 \pm 8.5$ & $8.4 \pm 8.0$ & 0.659 \\
\hline FBG $(\mathrm{mg} / \mathrm{dL})$ & $91.0 \pm 9.6$ & $88.4 \pm 11.1$ & $89.1 \pm 7.9$ & 0.782 \\
\hline BUN (mg/dL) & $26.1 \pm 4.0$ & $25.8 \pm 6.4$ & $25.5 \pm 8.4$ & 0.850 \\
\hline Creatinine (mg/dL) & $0.68 \pm 0.09$ & $0.69 \pm 0.12$ & $0.65 \pm 0.14$ & 0.212 \\
\hline $\operatorname{eGFR}\left(\mathrm{mL} / \mathrm{min} / 1.73 \mathrm{~m}^{2}\right)$ & $127.8 \pm 20.5$ & $135.4 \pm 36.2$ & $136.3 \pm 30.9$ & 0.917 \\
\hline $\mathrm{TC}(\mathrm{mg} / \mathrm{dL})$ & $195.6 \pm 28.1$ & $198.1 \pm 50.0$ & $190.0 \pm 47.8$ & 0.495 \\
\hline LDL-C (mg/dL) & $110.8 \pm 28.8$ & $114.3 \pm 39.7$ & $107.3 \pm 43.1$ & 0.490 \\
\hline HDL-C (mg/dL) & $50.4 \pm 16.9$ & $58.7 \pm 16.0$ & $62.9 \pm 18.9$ & 0.326 \\
\hline $\mathrm{TG}(\mathrm{mg} / \mathrm{dL})$ & $175.3 \pm 83.4$ & $123.5 \pm 62.8$ & $96.8 \pm 38.0$ & 0.038 \\
\hline $\mathrm{ESR}(\mathrm{mm})$ & $3.27 \pm 1.8$ & $20.0 \pm 12.6$ & $26.6 \pm 13.9$ & 0.041 \\
\hline $\mathrm{CRP}(\mathrm{mg} / \mathrm{dL})$ & $0.57 \pm 0.24$ & $1.56 \pm 0.26$ & $1.99 \pm 0.25$ & 0.490 \\
\hline \multicolumn{5}{|l|}{ Medication } \\
\hline Salazopyrin, n (\%) & - & $18(52.9)$ & $13(36.1)$ & \\
\hline Plaquenil, n (\%) & - & $20(55.5)$ & $28(77.7)$ & \\
\hline Steroid, n (\%) & - & $18(52.9)$ & $20(55.5)$ & \\
\hline Methotrexate, n (\%) & - & $19(55.8)$ & $29(80.5)$ & \\
\hline Leflunomide, n (\%) & - & $5(13.8)$ & $1(2.7)$ & \\
\hline Biologic therapya, n (\%) & - & $0(0)$ & $2(5.5)$ & \\
\hline DAS-28 & - & $2.29 \pm 0.63$ & $4.39 \pm 0.79$ & $<0.001$ \\
\hline
\end{tabular}

a: Tocilizumab: 1 patient, Rituximab: 1 patient. BMI: Body mass index, BUN: Blood urea nitrogen, CRP: reactive protein, DAS-28: Disease activity score, DBP: Diastolic blood pressure, eGFR: Estimated glomerular filtration rate, ESR: Erythrocyte sedimentation rate, HDL-C: High-density lipoprotein cholesterol, TG:Total Triglyceride LDL-C: Low-density lipoprotein cholesterol, MBP: Mean blood pressure, PP: Pulse pressure, SBP: Systolic blood pressure, TC: Total cholesterol. Data are expressed as mean \pm standard deviation or percentages as appropriate. 
was measured at the far vascular wall $1 \mathrm{~cm}$ proximal to the bifurcation of the CCA and the mean right and left CCA diameters were calculated. A QAS assessment was performed by entering the systolic and diastolic BP values into the Carotid Studio software. Cross-sectional distensibility coefficient (DC), CS and PWV values were automatically obtained by the software using the MoensKorteweg equation.

\section{Statistical analysis}

The clinical characteristics and CS parameters of the study population were statistically analyzed using the SPSS software version 20.0 (IBM corporation, Armonk, $\mathrm{NY}$ ). The data were expressed as mean $\pm \mathrm{SD}$ and analyzed using the Student t-test. A receiver operating characteristic (ROC) curve analysis was used to identify the optimal cut-off value for carotid PWV to identify maximum sensitivity and specificity for the evaluation of the increased AS. A multi-variate logistic regression analysis was undertaken to evaluate the impact of clinical and biochemical factors on local CS parameters. For all the tests, $\mathrm{p}<0.05$ was considered as statistically significant and $\mathrm{p}<0.001$ as significantly different.

\section{Results}

The clinical and demographic characteristics of the three groups (control, active RA, and inactive RA) are presented in Table I. No significant difference was observed between the groups in terms of age, BMI, BP, heart rate, disease duration, eGFR and FBG, BUN, creatinine, CRP, TC, HDL-C, and LDL-C levels ( $p>0.05$ for all). ESR was determined to be higher among the active RA patients compared to those with inactive RA (26.6 $\pm 13.9 \mathrm{~mm} v s 20.0 \pm 12.6 \mathrm{~mm}$, respectively, $\mathrm{p}=0.041$ ) and additionally active, inactive RA patients compared with control subject. In addition, CRP was significantly higher in both RA groups than the control group. When we assessed the lipid profiles of the patients, we observed significantly elevated TG levels in the inactive RA group compared with the active RA group and the TG levels of both groups were significantly higher than that of the control group. Lastly, the HDL-C levels were higher in the active RA group compared with the inactive group.

The local CS parameters of the three groups are presented in Table II. Carotid PWV was found to be significantly higher in the active RA patients compared to the inactive RA group and was also higher in both RA groups compared with the control group. In addition, the active RA group had significantly higher C-IMT values compared to the inactive RA group. Both RA groups had significantly higher C-IMT values compared with the control group. Furthermore, comparison between control group and the other groups showed that carotid diameters were significantly higher in the control group than in the RA groups; however, carotid distension was significantly higher only in the active RA group compared with the control group. Although carotid diameters were similar in the two RA groups ( $\mathrm{p}=0.454)$, carotid distension $(\mathrm{p}=0.012)$, distensibility $(\mathrm{p}=0.003)$, carotid PWV $(p=0.009)$, and PP $(p=<0.001)$ were significantly higher in the active RA group compared to the inactive RA group.

Table III demonstrates the correlation between the clinical characteristics and local CS of the RA patients. There was a positive correlation between CS and age, systolic BP, diastolic BP, mean BP, brachial PP, mean carotid PP, ESR and DAS-28. Table III also shows the correlation between the clinical characteristics and C-IMT in the RA patients. According to these results, a positive correlation was observed between C-IMT and age, BMI, systolic BP, brachial PP, mean carotid PP, TC, LDL-C, CRP level, and DAS-28. We did not find any statistically significant correlation between disease duration with CS and C-IMT.

A multivariate logistic regression analysis was performed to identify the factors that were related to local CS (Table IV). As a result, we determined age, systolic BP, brachial PP, carotid PP, ESR, and DAS-28 as independent predictors of CS.

For the evaluation of the increased AS, a ROC analysis was performed and provided the cut-off values for carotid PWV as $7.94 \pm 1.16 \mathrm{~m} / \mathrm{s}$ (sensitivity: $72 \%$ [95 \% CI 46-92\%], specificity: 64\% [95\%CI 54-88\%], positive predictive value: $68 \%$ [95\%CI 32-86\%], and negative predictive value: $66 \%$ [95\%CI 29-84\%] (AUC $=0.626$, $95 \% \mathrm{CI}=0.688-1.14, \mathrm{p}=0.012$ ).

\section{Discussions}

In this prospective study, we assessed the inter-relationship of local CS and C-IMT with disease activity in RA patients without traditional CV risk factors. Carotid PP, diameter, distension, distensibility, and PWV were utilized to estimate local CS. It was found that the active and inactive RA patients had statistically different local CS parameters and C-IMT.

Being an important cause of mortality in patients with RA, CV diseases have different manifestations. It has been shown that RA causes a 3-fold increase in the carotid atherosclerosis risk in patients with a similar risk profile, and is not only affected by traditional risk factors [13]. In RA, endothelial dysfunction caused by inflammatory processes increases the risk of $\mathrm{CV}$ diseases. In their prospective study, Zhang et al analyzed the effect of 
Table II. Carotid stiffness parameters in the study population.

\begin{tabular}{lllll}
\hline Arterial parameters & Controls $(\mathbf{n}=\mathbf{3 5})$ & Inactive RA group 1 $(\mathbf{n}=\mathbf{3 4})$ & Active RA group $(\mathbf{n}=\mathbf{3 6})$ & $\mathbf{p}$ value \\
\hline Carotid PP (mmHg) & $40.8 \pm 12.0$ & $36.6 \pm 8.76$ no space & $46.2 \pm 11.6$ & $<0.001$ \\
Carotid diameter (mm) & $8.28 \pm 1.0$ & $7.46 \pm 0.73$ & $7.60 \pm 0.83$ & 0.454 \\
Carotid distension (mm) & $0.32 \pm 0.12$ & $0.32 \pm 0.11$ & $0.39 \pm 0.11$ & $\mathbf{0 . 0 1 2}$ \\
Carotid distensibility (kPa-1 $10-3)$ & $25.6 \pm 12.8$ & $19.1 \pm 8.6$ & $26.6 \pm 11.7$ & $\mathbf{0 . 0 0 3}$ \\
C-IMT (mm) & $0.68 \pm 0.12$ & $0.60 \pm 0.12$ & $0.68 \pm 0.15$ & $\mathbf{0 . 0 1 5}$ \\
Carotid PWV (m/s) & $6.41 \pm 0.98$ & $7.32 \pm 1.19$ & $8.20 \pm 1.47$ & $\mathbf{0 . 0 0 9}$ \\
\hline
\end{tabular}

PP: Pulse pressure, C-IMT: carotid intima-media thickness PWV: Pulse wave velocity. Data are expressed as mean \pm SD.

Table III. Carotid stiffness and carotid intima-media thickness correlation with clinical characteristics in the rheumatoid arthritis population $(\mathrm{n}=70)$.

\begin{tabular}{|c|c|c|c|c|}
\hline \multirow[t]{2}{*}{ Risk factors } & \multicolumn{2}{|c|}{ Carotid stiffness (pulse wave velocity) } & \multicolumn{2}{|c|}{ Carotid intima-media thickness } \\
\hline & $\mathbf{r}$ & p & $\mathbf{r}$ & p \\
\hline Age & 0.435 & $<0.001$ & 0.676 & $<0.001$ \\
\hline BMI & 0.258 & 0.06 & 0.294 & 0.031 \\
\hline Disease duration & 0.177 & 0.200 & 0.029 & 0.836 \\
\hline Systolic BP & 0.494 & $<0.001$ & 0.292 & 0.015 \\
\hline Diastolic BP & 0.242 & 0.045 & 0.169 & 0.166 \\
\hline Brachial PP & 0.511 & $<0.001$ & 0.267 & 0.026 \\
\hline Mean BP & 0.355 & 0.003 & 0.227 & 0.061 \\
\hline Mean carotid PP & 0.631 & $<0.001$ & 0.249 & 0.039 \\
\hline $\mathrm{TC}$ & 0.132 & 0.287 & 0.251 & 0.040 \\
\hline HDL-C & -0.212 & 0.085 & 0.013 & 0.916 \\
\hline LDL-C & 0.207 & 0.093 & 0.281 & 0.021 \\
\hline TG & 0.148 & 0.232 & 0.036 & 0.773 \\
\hline eGFR & 0.109 & 0.433 & 0.258 & 0.060 \\
\hline ESR & 0.257 & 0.033 & 0.227 & 0.060 \\
\hline CRP & 0.016 & 0.897 & 0.311 & 0.009 \\
\hline DAS-28 & 0.314 & 0.009 & 0.292 & 0.015 \\
\hline C-IMT & 0.223 & 0.066 & - & - \\
\hline
\end{tabular}

BMI: Body mass index, BP: Blood pressure, PP: Pulse pressure, TC: Total cholesterol, HDL-C: High-density lipoprotein cholesterol, LDLC: Low-density lipoprotein cholesterol, TG: Triglyceride eGFR: Estimated glomerular filtration rate, ESR: Erythrocyte sedimentation rate, CRP:C-reactive protein, DAS-28: Disease activity score-28, C-IMT: carotid intima-media thickness

Table IV. Multivariate logistic regression analysis of factors related to local carotid AS

\begin{tabular}{llll}
\hline & Odds ratio & $\mathbf{9 5 \%}$ confidence interval & P value \\
\hline Age & 0.069 & $0.034-0.104$ & $<\mathbf{0 . 0 0 1}$ \\
BMI & 0.079 & $-0.003-0.162$ & 0.060 \\
Systolic BP & 0.041 & $0.024-0.059$ & $<\mathbf{0 . 0 0 1}$ \\
Diastolic BP & 0.025 & $0.003-0.046$ & $\mathbf{0 . 0 2 4}$ \\
Mean BP & 0.018 & $-0.001-0.038$ & 0.066 \\
Brachial PP & 0.073 & $0.044-0.102$ & $<\mathbf{0 . 0 0 1}$ \\
Carotid PP & 0.096 & $0.064-0.129$ & $<\mathbf{0 . 0 0 1}$ \\
Disease duration & 0.031 & $-0.017-0.080$ & 0.200 \\
TC & 0.005 & $-0.004-0.013$ & 0.287 \\
LDL-C & 0.008 & $-0.003-0.020$ & 0.159 \\
HDL-C & -0.020 & $-0.044-0.005$ & 0.119 \\
TG & 0.000 & $-0.010-0.009$ & 0.944 \\
eGFR & -0.007 & $-0.022-0.008$ & 0.376 \\
ESR & 0.028 & $0.003-0.053$ & 0.027 \\
CRP & -0.001 & $-0.002-0.001$ & 0.430 \\
DAS-28 & 0.259 & $0.001-0.517$ & 0.022 \\
C-IMT & 0.002 & $-0.001-0.005$ & 0.249 \\
\hline
\end{tabular}

BMI: body mass index, BP: blood pressure, PP: pulse pressure, TC: Total Cholesterol, LDL-C: low-density lipoprotein, HDL-C: high-density lipoprotein, TG: Triglyceride, eGFR: estimated glomerular filtration rate, ESR: erythrocyte sedimentation rate, CRP: C-reactive protein, DAS-28: disease activity score-28, C-IMT: Carotid intima-media thickness. 
diabetes mellitus on the endothelial function and found no correlation with C-IMT but local CS showed a significant correlation with diabetes mellitus [14]. In this study, the discrepancy between stiffness and C-IMT was attributed to the hypothesis that endothelial dysfunction is a precursor of atherosclerosis. Since the endothelial dysfunction is accepted as an indicator of an atherosclerotic process, its assessment is essential to predict CV risk in RA. CV risk assessment from carotid artery is frequently evaluated using manual C-IMT measurement. However, manual measurement is highly dependent on the capability of human eye to differentiate between the layer interfaces, and is affected by several other factors such as the sonographer's experience and hand sensitivity in positioning the electronic calipers and ultrasound settings [10,15-17]. Furthermore, previous studies have shown that semi-automated C-IMT measurements reduce interreader variability [18]. Therefore, a novel RF-based ultrasound technique has been proposed to provide an automated assessment of C-IMT and local CS in one session. Local CS evaluation by this method also allows the easy evaluation of endothelial function. Additionally, although C-IMT is conventionally used as a marker of subclinical atherosclerosis, Spence et al showed that it is primarily affected by age and blood pressure. The authors also suggested that increased C-IMT might essentially indicate hypertensive medial hypertrophy or smooth muscle thickening in the media layer rather than IMT thickening that characterizes atherosclerosis [19]. These findings also clearly demonstrate the necessity of a new practical technique to evaluate the atherosclerotic burden in addition to C-IMT. In the present study, we measured local stiffness and C-IMT of the RA patients at the carotid artery using the RF-based ultrasound technique.

We found that the active RA group had a significantly higher C-IMT compared to the inactive group. Schreuder et al demonstrated that the C-IMT values measured with $\mathrm{RF}$ and B-mode from the CCA had similar reproducibility and exhibited an acceptable correlation [20]. Furthermore, Naredo et al evaluated the multi-examiner interobserver and intra-observer reliability for QIMT and they found good to excellent inter-observer reliability and good to optimal intra-observer reliability [21]. Similarly, when we evaluated the relationship between C-IMT and clinical characteristics, the results of the meta-analysis showed that CRP was associated with higher C-IMT and no correlation was found between C-IMT and disease duration [22]. Furthermore, in addition to the correlation between C-IMT with CRP and DAS-28, Lee et al also reported a correlation with disease duration [23].

Our results are in line with those reported in earlier studies that demonstrated a correlation between carotid to femoral PWV and a higher disease activity score [24]. Among the studied parameters, the age, ESR, and DAS28 had a positive correlation with carotid PWV probably due to the endothelium having been affected by chronic inflammation. Other studies have reported that aortic PWV was correlated with elevated CRP, ESR, and DAS28 [14]. Age and DAS-28 have also been previously determined as independent predictors of AS in RA patients $[25,9]$. Lee et al evaluated local CS in RA patients by speckle tracking strain imaging and found that local CS had a significant correlation with age, disease duration, and DAS-28 [23]. Similarly, in our study population, age, DAS-28, and ESR were the independent predictors of local CS according to the results of the multivariate logistic regression analysis.

Del Rincon et al showed that the CV risk in RA patients increased with disease duration with the effect being significantly greater after 20 years [26]. In our study, we also evaluated the correlation between local CS parameters and disease duration but no positive correlation was found. This discrepancy may be attributed to the relatively shorter disease duration of our patients with only 4 having had RA for longer than 20 years. Kaisa et al demonstrated that AS parameters could be reduced by anti-inflammatory therapy [27]. In the current study, we could not examine the effect of medical treatment on patients since our patients were on heterogeneous medication and the number of patients who met the inclusion criteria of a longitudinal study was low. In addition, the use of heterogeneous medication was one of the limitations of our study since we were not able to assess the effect of the drugs. The last limitation of the study concerns the limited number of patients and the control subjects.

Despite the limitations, the current study is significant in terms of being the first in the literature to evaluate $\mathrm{C}$ IMT and PWV via a novel ultrasound-based technique. Our results are consistent with those reported in previous studies that have used applanation tonometry [24]. These results provide evidence for the effectiveness and applicability of this novel technique. Furthermore, being easy-to-use and feasible, this technique also allows the evaluation of broad patient populations such as those with RA, who require additional information to predict the $\mathrm{CV}$ risk.

\section{Conclusions}

Despite the limited number of the patients in this study, we obtained useful information concerning the CV risk status of RA patients. Further studies are required to support our findings and shed light on the relationship of local CS with disease duration and medication. This 
easily applicable and previously confirmed method can be used in future to access the CV risk in broader study populations with different $\mathrm{CV}$ risks.

\section{Conflict of interest: none}

\section{References}

1. Alamanos Y, Drosos AA. Epidemiology of adult rheumatoid arthritis. Autoimmun Rev 2005;4:130-136.

2. Gibofsky A. Overview of epidemiology, pathophysiology and diagnosis of rheumatoid arthritis. Am J Manag Care 2012;18:S295-S302.

3. Wolfe F, Mitchell DM, Sibley JT, et al. The mortality of rheumatoid arthritis. Arthritis Rheum 1994;37:481-494.

4. Symmons DP, Jones MA, Scott DL, Prior P. Long term mortality outcome in patients with rheumatoid arthritis: early presenters continue to do well. J Rheumatol 1998;25:1072-1077.

5. Van Doornum S, McColl G, Wicks IP. Accelerated atherosclerosis: an extra articular feature of rheumatoid arthritis? Arthritis Rheum 2002;46:862-873.

6. Libby P. Role of inflammation in atherosclerosis associated with rheumatoid arthritis. Am J Med 2008;121:S21-S31.

7. Lüscher TF, Barton M. Biology of the endothelium. Clin Cardiol 1997;20:II3-II10.

8. Nurmohamed MT, Herslinga M, Kitas GD. Cardiovascular comorbidity in rheumatic diseases. Nat Rev Rheumatol 2015;11:693-704.

9. Ambrosino P, Tasso M, Lupoli R, et al. Non-invasive assessment of arterial stiffness in patients with rheumatoid arthritis: a systematic review and meta analysis of literature studies. Ann Med 2015;47:457-467.

10. Flore R, Ponziani FR, Tinelli G, et al. New modalities of ultrasound-based intima-media thickness, arterial stiffness and non-coronary vascular calcifications detection to assess cardiovascular risk. Eur Rev Med Pharmacol Sci 2015;19:1430-1441.

11. Luo J, Li RX, Konofagou EE. Pulse wave imaging of the human carotid artery: an in vivo feasibility study. IEEE Trans Ultrason Ferroelectr Freq Control 2012;59:174-181.

12. Giannarelli C, Bianchini E, Bruno RM, et al. Local carotid stiffness and intima-media thickness assessment by a novel ultrasound-based system in essential hypertension. Atherosclerosis 2012;223:372-377.

13. Roman MJ, Salmon JE. Cardiovascular manifestations of rheumatologic diseases. Circulation 2007;116:2346-2355.

14. Zhang L, Yin JK, Duan YY, et al. Evaluation of carotid artery elasticity changes in patients with type 2 diabetes. Cardiovasc Diabetol 2014;13:39.

15. Mäki-Petäjä KM, Elkhawad M, Cheriyan J, et al. Anti-tumor necrosis factor-a therapy reduces aortic inflammation and stiffness in patients with rheumatoid arthritis. Circulation 2012; 126:2473-2480.

16. Wendelhag I, Liang Q, Gustavsson T, Wikstrand J. A new automated computerized analyzing system simplifies readings and reduces the variability in ultrasound measurement of intima-media thickness. Stroke 1997;28:2195-2200.

17. Seçil M, Altay C, Gülcü A, Ceçe H, Göktay AY, Dicle O. Automated measurement of intima-media thickness of carotid arteries in ultrasonography by computer software. Diagn Interv Radiol 2005;11:105-108.

18. Dogan S, Duivenvoorden R, Grobbee DE, et al. Ultrasound protocols to measure carotid intima-media thickness in trials; comparison of reproducibility, rate of progression, and effect of intervention in subjects with familial hypercholesterolemia and subjects with mixed dyslipidemia. Ann Med 2010;42:447-464.

19. Spence JD. Technology insight: ultrasound measurement of carotid plaque--patient management, genetic research, and therapy evaluation. Nat Clin Pract Neurol 2006;2:611-619.

20. Schreuder FH, Graf M, Hameleers JM, Mess WH, Hoeks AP. Measurement of common carotid artery intimamedia thickness in clinical practice: comparison of Bmode and RF-based technique. Ultraschall Med 2009;5:459465 .

21. Naredo E1, Möller I, Gutiérrez M, et al. Multi-examiner reliability of automated radio frequency-based ultrasound measurements of common carotid intima-media thickness in rheumatoid arthritis. Rheumatology 2011;50:18601864.

22. Ambrosino P, Lupoli R, Di Minno A, Tasso M, Peluso R, Di Minno MN. Subclinical atherosclerosis in patients with rheumatoid arthritis. A meta-analysis of literature studies. Thromb Haemost 2015;113:916-930.

23. Lee JH, Cho K, Kim SM. Carotid arterial stiffness in patients with rheumatoid arthritis assessed by speckle tracking strain imaging: its association with carotid atherosclerosis. Clin Exp Rheumatol 2012;30:720-728.

24. Botta E, Meroño T, Saucedo C, et al. Associations between disease activity, markers of HDL functionality and arterial stiffness in patients with rheumatoid arthritis. Atherosclerosis 2016;251:438-444.

25. Kocabay G, Hasdemir H, Yildiz M. Evaluation of pulse wave velocity in systemic lupus erythematosus, rheumatoid arthritis and Behcet's disease. J Cardiol 2012;59:72-77.

26. Del Rincon I, O'Leary DH, Freeman GL, Escalante A. Acceleration of atherosclerosis during the course of rheumatoid arthritis. Atherosclerosis 2007;195:354-360.

27. DiGeso L, Zardi EM, Afeltra A, et al. Comparison between conventional and automated software-guided ultrasound assessment of bilateral common carotid intima-media thickness in patients with rheumatic diseases. Clin Rheumatol 2012;31:881-884. 